\title{
Boundedness and Asymptotic Behaviour of the Solutions for a Third - Order Fuzzy Difference Equation
}

\author{
Xiaotong Jing ${ }^{1}$, Yonghong $\mathrm{Li}^{1,}$, , Changyou Wang ${ }^{1,2, *}$ \\ ${ }^{1}$ College of Science, Chongqing University of Posts and Telecommunications, Chongqing, P. R. China \\ ${ }^{2}$ College of Applied Mathematics, Chengdu University of Information Technology, Chengdu, P. R. China
}

\section{Email address:}

1194330567@qq.com (Xiaotong Jing), liyh@cqupt.edu.cn (Yonghong Li),wangcy@cuit.edu.cn (Changyou Wang)

*Corresponding author

\section{To cite this article:}

Xiaotong Jing, Yonghong Li, Changyou Wang. Boundedness and Asymptotic Behaviour of the Solutions for a Third - Order Fuzzy Difference Equation. Internet of Things and Cloud Computing. Vol. 7, No. 1, 2019, pp. 1-11. doi: 10.11648/j.iotcc.20190701.11

Received: December 21, 2018; Accepted: January 14, 2019; Published: January 30, 2019

\begin{abstract}
Our aim in this paper is to investigate the dynamics of a third-order fuzzy difference equation. By using new iteration method for the more general nonlinear difference equations and inequality skills as well as a comparison theorem for the fuzzy difference equation, some sufficient conditions which guarantee the existence, unstability and global asymptotic stability of the equilibriums for the nonlinear fuzzy system are obtained. Moreover, some numerical solutions of the equation describing the system are given to verify our theoretical results.
\end{abstract}

Keywords: Fuzzy Difference Equation, Boundedness, Existence, Uniqueness, Asymptotic Behavior

\section{Introduction}

Nonlinear difference equation is an important mathematical models which describe the relationship between the real world phenomenons. It not only enriched the theory of mathematics, but also solved the practical problem, such as the fields of the number of population structure analysis, economic, genetic, biology etc. (see, eg., [1-5] and the references therein). In recent years, research on discrete systems has become a hot problem. (see, eg., $[6,7]$ ).

However, the traditional mathematics theories and methods seem to be inadequate in the face of fuzzy phenomenon. Based on this background, the fuzzy difference systems are a powerful tool which can be used to study better some uncertain phenomenons. In recent decades, the fuzzy mathematics theory and its applications have achieved fruitful results. In view of the fact, the fuzzy difference equation system has attracted more and more interest which further enriches the research of the difference system.

In 1998, DeVault et al [8] discussed the existence, boundedness, oscillation behavior of the positive solutions and the global asymptotic behavior of the equilibrium points for the nonlinear difference equation.

$$
x_{n+1}=A+\frac{x_{n}}{x_{n-1}}, n=0,1, \ldots
$$

Where $A, x_{-1}, x_{0}$ are positive numbers.

Similarly, In 1998, Papaschinopoulos and Schinas [9] studied the following difference equations.

$$
x_{n+1}=A+\frac{y_{n}}{x_{n-p}}, y_{n+1}=A+\frac{x_{n}}{y_{n-p}}, n=0,1, \ldots,
$$

Where $A, x_{-p}, x_{-p+1}, \ldots, x_{0}, y_{-q}, y_{-q+1}, \ldots, y_{0}$ are positive real numbers $p, q$ are positive integers. There are similar conclusions that if $p=q=1, x_{n}=y_{n}, n=-1,0, \ldots$, then $x_{n}$ is both a solution of Eq. (2) and the solution of Eq. (1).

Besides, Zhang et al [10] researched the following nonlinear fuzzy difference equation.

$$
x_{n+1}=\frac{a+b x_{n}}{A+x_{n-1}}, n=0,1,2, \ldots,
$$

Where $\left\{x_{n}\right\}$ is a sequence of positive fuzzy number, $a, b, A$ and the initial values $x_{-1}, x_{0}$ are positive fuzzy 
numbers. The existence and boundedness of the positive solutions asymptotic behavior of the equilibrium points for the difference equation are discussed. Moreover, In 2014, Zhang et al [11] continuously proved similar conclusion for the follow first-order fuzzy difference equation

$$
x_{n+1}=\frac{A+x_{n}}{B+x_{n}}, n=0,1,2, \ldots,
$$

Where $\left\{x_{n}\right\}$ is a sequence of positive fuzzy numbers, $A, B$ and the initial value $x_{0}$ are positive fuzzy numbers.

More recently, Wang et al [12] investigate the existence and uniqueness of the positive solutions and the asymptotic behavior of the equilibrium points of the following fuzzy difference equation.

$$
x_{n+1}=\frac{A x_{n-1} x_{n-2}}{D+B x_{n-3}+C x_{n-4}}, n=0,1,2, \ldots,
$$

Where $\left\{x_{n}\right\}$ is a sequence of positive fuzzy numbers, the parameters $A, B, C, D$ and the initial conditions $x_{-4}, x_{-3}, x_{-2}, x_{-1}, x_{0}$ are positive fuzzy numbers. For closely related papers in this research area, see, for example, [13-30] and the references therein.

Motivated by the discussions above, the purpose of this paper is to discuss the existence and uniqueness of the positive solutions and the asymptotic behavior of the equilibrium points for the following third-order fuzzy difference equation.

$$
x_{n+1}=\frac{A x_{n-2}}{B+C x_{n-2} x_{n-1} x_{n}}, n=0,1,2, \ldots,
$$

Where $\left\{x_{n}\right\}$ is a sequence of positive fuzzy numbers, the parameters $A, B, C$ and the initial conditions $x_{-2}, x_{-1}, x_{0}$ are positive fuzzy numbers.

This paper is arranged as follows: in Section 2, some definitions and preliminary results are given. The main results and related proofs are obtained in Section 3. Finally, some numerical examples are used to illustrate our theoretical results.

\section{Preliminaries and Notations}

For the convenience of readers, some definitions and preliminary results related to the theoretical proof of this paper are given, see [31-35].

Definition 2.1 For a set $B$ we denote by $\bar{B}$ the closure of $B$. We say that a function $A: R \rightarrow[0,1]$ is a fuzzy number if the follow conclusions are true:

(i) $A$ is normal, i.e., there exists $x \in R$ such that $A(x)=1$;

(ii) $A$ is a fuzzy convex set, i.e., $A\left(t x_{1}+(1-t) x_{2}\right) \geq \min \left\{A\left(x_{1}\right), A\left(x_{2}\right)\right\}, \forall t \in[0,1], x_{1}, x_{2} \in R$;

(iii) $A$ is upper semicontinuous on $R$; (iv) The support of $A$, i.e., supp $A=\overline{\cup_{\alpha \in(0,1]}[A]_{\alpha}}=\overline{\{x \in R: A(x)>0\}}$ is compact.

where the $\alpha$-cuts of $A$ are closed intervals, define as $[A]_{\alpha}=\{x \in R: A(x) \geq \alpha$,$\} if supp A$

$\subset(0, \infty)$ then fuzzy number $A$ is obviously positive.

Definition 2.2 Let $A, B$ be fuzzy numbers which satisfy $[A]_{\alpha}=\left[A_{l, \alpha}, A_{r, \alpha}\right],[B]_{\alpha}=\left[B_{l, \alpha}, B_{r, \alpha}\right], \quad \alpha \in(0,1]$, the follow metric is denoted

$$
D(A, B)=\sup \max \left\{\left|A_{l, \alpha}-B_{l, \alpha}\right|,\left|A_{r, \alpha}-B_{r, \alpha}\right|\right\},
$$

Where sup is taken for all $\alpha \in(0,1]$. Then $\left(R_{f}, D\right)$ is a complete metric space. For the convenience of application in the future, we define $\hat{0} \in R_{f}$ as

$$
\hat{0}(x)=\left\{\begin{array}{l}
1, x=0 \\
0, x \neq 0
\end{array}\right.
$$

Thus, $[\hat{0}]_{\alpha}=[0,0], 0<\alpha<1$.

Definition 2.3 Persistence (resp. boundedness) of fuzzy numbers is defined, if there exist positive real number $M(\operatorname{resp} . N)$, such that the following conclusions are true $\sup p x_{n} \subset[M, \infty]\left(\operatorname{resp} \cdot \sup p x_{n} \subset(0,1]\right), n=1,2, \ldots$, where $\left\{x_{n}\right\}$ is a sequence of positive fuzzy numbers.

Further, $\left\{x_{n}\right\}$ is bounded and persistent if there exist positive real numbers $M, N$, such that $\sup p x_{n} \subset[M, N], n=1,2, \ldots$.

Lemma 2.1 Let $I_{x}, I_{y}$ be some intervals of real numbers and let $f: I_{x}^{k+1} \times I_{y}^{l+1} \rightarrow I_{x}, \quad g: I_{x}^{k+1} \times I_{y}^{l+1} \rightarrow I_{y} \quad$ be continuously differential functions. Then for every set of initial conditions $\left(x_{i}, y_{j}\right) \in I_{x} \times I_{y},(i=-k,-k+1, \ldots, 0, j=-l,-l+1, \ldots, 0)$, the following system of difference equations

$$
\left\{\begin{array}{l}
x_{n+1}=f\left(x_{n}, x_{n-1}, \ldots, x_{n-k}, y_{n}, y_{n-1}, \ldots, y_{n-l}\right), \\
y_{n+1}=g\left(x_{n}, x_{n-1}, \ldots, x_{n-k}, y_{n}, y_{n-1}, \ldots, y_{n-l}\right)
\end{array}\right.
$$

Has a unique solution $\left\{\left(x_{i}, y_{j}\right)\right\}_{i=-k, j=-l}^{+\infty,+\infty}$.

Definition 2.4 A point $(\bar{x}, \bar{y}) \in I_{x} \times I_{y}$ is called an equilibrium point of system (7) if $\bar{x}=f(\bar{x}, \bar{x}, \ldots, \bar{x}$, $\bar{y}, \bar{y}, \ldots, \bar{y}), \quad \bar{y}=g(\bar{x}, \bar{x}, \ldots, \bar{x}, \bar{y}, \bar{y}, \ldots, \bar{y}), \quad$ that is, $\left(x_{n}, y_{n}\right)=(\bar{x}, \bar{y})$ for $n \geq 0$ is the solution of difference system (7), or equivalently, $(\bar{x}, \bar{y})$ is a fixed point of the vector map $(f, g)$.

Definition 2.5 Suppose that $(\bar{x}, \bar{y})$ be an equilibrium point of the system (7), then we have 
(i) $(\bar{x}, \bar{y})$ is called locally stable if for every $(\bar{x}, \bar{y})$ there exists $\delta>0$ such that for any initial conditions $\left(x_{i}, y_{i}\right) \in I_{x} \times I_{y}(i=-k, \ldots, 0, j=-l, \ldots, 0)$ with $\sum_{i=-k}^{0}\left|x_{i}-\bar{x}\right|<\delta, \sum_{j=-l}^{0}\left|y_{i}-\bar{y}\right|<\delta$, we have $\left|x_{n}-\bar{x}\right|<\varepsilon$, $\left|y_{n}-\bar{y}\right|<\varepsilon$ for any $n>0$.

(ii) $(\bar{x}, \bar{y})$ is called attractor if $\lim _{n \rightarrow \infty} x_{n}=\bar{x}, \lim _{n \rightarrow \infty} y_{n}=\bar{y}$, for any initial conditions $\left(x_{i}, y_{j}\right) \in I_{x} \times I_{y}$, $(i=-k,-k+1, \ldots, 0, j=-l,-l+1, \ldots, 0)$.

(iii) $(\bar{x}, \bar{y})$ is called asymptotically stable if it is stable, and $(\bar{x}, \bar{y})$ is also attractor.

(iv) $(\bar{x}, \bar{y})$ is called unstable if it is not locally stable.

Definition 2.6 Let $(\bar{x}, \bar{y})$ be an equilibrium point of the vector map $F=\left(f, x_{n}, \ldots, x_{n-k}, g, y_{n}, \ldots, y_{n-l}\right)$, where $f$ and $g$ are continuously differential functions at $(\bar{x}, \bar{y})$ The linearized system of (7) about the equilibrium point $(\bar{x}, \bar{y})$ is $X_{n+1}=F\left(X_{n}\right)=F_{J} \cdot X_{n}$, where $F_{J}$ is the Jacobian matrix of the system (7) about $(\bar{x}, \bar{y})$ and $X_{n}=\left(x_{n}, \ldots, x_{n-k}, y_{n}, \ldots, y_{n-l}\right)^{T}$.

Definition 2.7 let $p, q, s, t$ be four nonnegative integers such that $p+q=n, s+t=m$, splitting $x=\left(x_{1}, x_{2} \ldots, x_{n}\right)$ into $x=\left([x]_{p},[x]_{q}\right)$ and $y=\left(y_{1}, y_{2} \ldots, y_{m}\right)$ into $y=\left([y]_{S},[y]_{t}\right)$, where $[x]_{\sigma}$ denotes a vector with $\sigma$-components of $x$. We say that the function $f=\left(x_{1}, x_{2} \ldots, x_{n}, y_{1}, y_{2} \ldots, y_{m}\right)$ possesses a mixed monotone property in subsets $I_{x}^{n} \times I_{y}^{m}$ of $R^{n} \times R^{m}$ if $f=\left([x]_{p},[x]_{q},[y]_{s},[y]_{t}\right)$ is monotone non-decreasing in each component of $\left([x]_{p},[y]_{S}\right)$, and is monotone non-increasing in each component of $\left([x]_{q},[y]_{t}\right)$ for $(x, y) \in I_{x}^{n} \times I_{y}^{m}$. In particular, if $q=0, t=0$, then it is said to be monotone non-decreasing in $I_{x}^{n} \times I_{y}^{m}$.

Lemma 2.2 Assume that $X_{n+1}=F\left(X_{n}\right), n=0,1, \ldots$, is a system of difference equations and $\bar{X}$ is the equilibrium point of this system, i.e. $F(\bar{X})=\bar{X}$. Then we have. (i) If all eigenvalues of the Jacobian matrix $J_{F}$ about $\bar{X}$ lie inside the open unit disk $|\lambda|<1$, then $\bar{X}$ is locally asymptotically stable.

(ii) If one of eigenvalues of the Jacobian $J_{F}$ matrix about $\bar{X}$ has norm greater than one, then $\bar{X}$ is unstable.

\section{Main Results}

The following lemmas are applied to study the existence and uniqueness of the positive solutions of equations (6).

Lemma 3.1 [36] Let $f$ be a continuous function from $R^{+} \times R^{+} \times R^{+} \rightarrow R^{+} \quad$ into $R^{+}$and $A, B, C$ be fuzzy numbers, then

$$
[f(A, B, C)]_{\alpha}=f\left([A]_{\alpha},[B]_{\alpha},[C]_{\alpha}\right), \alpha \in(0,1] .
$$

Lemma $3.2 \quad$ [37] Let $u \in R_{f} \quad$ write $[u]_{\alpha}=\left[u_{l, \alpha}, u_{r, \alpha}\right], \alpha \in(0,1]$. Then $u_{l, \alpha}$ and $u_{r, \alpha}$ can be regarded as functions on $(0,1]$ which satisfy

(i) $u_{l, \alpha}$ is nondecreasing and left continuous,

(ii) $u_{r, \alpha}$ is nonincreasing and left continuous,

(iii) $u_{l, \alpha} \leq u_{r, \alpha}$.

Conversely for any functions $a(\alpha)$ and $b(\alpha)$ defined on $(0,1]$ which satisfy the above (i)-(iii), there exists a unique $u \in R_{f}$ such that $u(\alpha)=[a(\alpha), b(\alpha)]$ for any $\alpha \in(0,1]$.

Theorem 3.1 Consider equation (6), where $A, B, C$ are positive fuzzy numbers. Then for any positive fuzzy numbers $x_{-2}, x_{-1}, x_{0}$, there exist a unique positive solution $x_{n}$ of Eq. (6).

Proof. Suppose that there exists a sequence of fuzzy numbers $\left\{x_{n}\right\}$ satisfying Eq. (6) with initial conditions $x_{-2}, x_{-1}, x_{0}$. Consider the $\alpha$-cuts, $\alpha \in(0,1]$,

$$
\begin{aligned}
& {[A]_{\alpha}=\left[A_{l, \alpha}, A_{r, \alpha}\right],[B]_{\alpha}=\left[B_{l, \alpha}, B_{r, \alpha}\right],} \\
& {[C]_{\alpha}=\left[C_{l, \alpha}, C_{r, \alpha}\right],\left[x_{n}\right]_{\alpha}=\left[L_{n, \alpha}, R_{n, \alpha}\right], n=-2,-1, \cdots .}
\end{aligned}
$$

The following results are true from (6), (8) and Lemma 3.1

$$
\begin{gathered}
{\left[x_{n+1}\right]_{\alpha}=\left[L_{n+1, \alpha}, R_{n+1, \alpha}\right]=\left[\frac{A x_{n-2}}{B+C x_{n-2} x_{n-1} x_{n}}\right]_{\alpha}=\frac{\left[A x_{n-2}\right]_{\alpha}}{\left[B+C x_{n-2} x_{n-1} x_{n}\right]_{\alpha}}} \\
=\frac{\left[A_{l, \alpha}, A_{r, \alpha}\right]\left[L_{n-2, \alpha}, R_{n-2, \alpha}\right]}{\left[B_{l, \alpha}, B_{r, \alpha}\right]+\left[C_{l, \alpha}, C_{r, \alpha}\right]\left[L_{n-2, \alpha}, R_{n-2, \alpha}\right]\left[L_{n-1, \alpha}, R_{n-1, \alpha}\right]\left[L_{n, \alpha}, R_{n, \alpha}\right]} \\
=\frac{\left[A_{l, \alpha} L_{n-2, \alpha}, A_{r, \alpha} R_{n-2, \alpha}\right]}{\left[B_{l, \alpha}+C_{l, \alpha} L_{n-2, \alpha} L_{n-1, \alpha} L_{n, \alpha}, B_{r, \alpha}+C_{r, \alpha} R_{n-2, \alpha} R_{n-1, \alpha} R_{n, \alpha}\right]}
\end{gathered}
$$




$$
=\left[\frac{A_{l, \alpha} L_{n-2, \alpha}}{B_{r, \alpha}+C_{r, \alpha} R_{n-2, \alpha} R_{n-1, \alpha} R_{n, \alpha}}, \frac{A_{r, \alpha} R_{n-2, \alpha}}{B_{l, \alpha}+C_{l, \alpha} L_{n-2, \alpha} L_{n-1, \alpha} L_{n, \alpha}}\right] .
$$

Hence, for $\alpha \in(0,1], n=-2,-1, \cdots$, according to the above result, it follows that

$$
\begin{aligned}
L_{n+1, \alpha} & =\frac{A_{l, \alpha} L_{n-2, \alpha}}{B_{r, \alpha}+C_{r, \alpha} R_{n-2, \alpha} R_{n-1, \alpha} R_{n, \alpha}}, \\
R_{n+1, \alpha} & =\frac{A_{r, \alpha} R_{n-2, \alpha}}{B_{l, \alpha}+C_{l, \alpha} L_{n-2, \alpha} L_{n-1, \alpha} L_{n, \alpha}} .
\end{aligned}
$$

Then, for any initial conditions $\left(L_{j, \alpha}, R_{j, \alpha}\right), j=-2,-1,0, \alpha \in(0,1]$, it is obvious from Lemma 2.1 that exists a unique solution $\left(L_{n, \alpha}, R_{n, \alpha}\right)$ of the systems (9).

$$
\begin{aligned}
& 0<A_{l, \alpha_{1}} \leq A_{l, \alpha_{2}} \leq A_{r, \alpha_{2}} \leq A_{r, \alpha_{1}}, 0<B_{l, \alpha_{1}} \leq B_{l, \alpha_{2}} \leq B_{r, \alpha_{2}} \leq B_{r, \alpha_{1}}, \\
& 0<C_{l, \alpha_{1}} \leq C_{l, \alpha_{2}} \leq C_{r, \alpha_{2}} \leq C_{r, \alpha_{1}}, 0<L_{j, \alpha_{1}} \leq L_{j, \alpha_{2}} \leq R_{j, \alpha_{2}} \leq R_{j, \alpha_{1}}, j=-2,-1,0 .
\end{aligned}
$$

Where $A, B, C, x_{j}(j=-2,-1,0)$ are positive fuzzy numbers.

Next, the following conclusions is proved to be true by mathematical induction.

$$
0<L_{n, \alpha_{1}} \leq L_{n, \alpha_{2}} \leq R_{n, \alpha_{2}} \leq R_{n, \alpha_{1}}, n=1,2, \cdots
$$

From (11), the conclusions (12) are true for $n=-2,-1,0$. Suppose that (12) are true for $n \leq k, k \in\{1,2, \cdots\}$, then from (9)-(12), it follows that for $n=k+1$.

Hence (12) are true

Thus, from (9), it holds that

$$
L_{1^{\prime}, \alpha}=\frac{A_{l, \alpha} L_{-2, \alpha}}{B_{r, \alpha}+C_{r, \alpha} R_{-2, \alpha} R_{-1, \alpha} R_{0, \alpha}}, R_{1, \alpha}=\frac{A_{r, \alpha} R_{-2, \alpha}}{B_{l, \alpha}+C_{l, \alpha} L_{-2, \alpha} L_{-1, \alpha} L_{0, \alpha}}, \alpha \in(0,1] .
$$

Moreover, in view of $A, B, C, x_{j},(j=-2,-1,0)$ are positive fuzzy numbers, then $A_{l, \alpha}, A_{r, \alpha}, B_{l, \alpha}, B_{r, \alpha} B_{l, \alpha}, C_{l, \alpha}$, $C_{r, \alpha}, L_{-2, \alpha}, R_{-2, \alpha}, L_{-1, \alpha}, R_{-1, \alpha}, L_{0, \alpha}, R_{0, \alpha}$ are left continuous from Lemma 3.2. So, it is obvious that $L_{1, \alpha}, R_{1, \alpha}$ are also left continuous from (13). Finally, it is obtained by mathematical induction that $L_{n, \alpha}, R_{n, \alpha}, n=1,2, \cdots$, are left continuous.

Now, we prove that the support of $x_{n}$, i.e., supp $x_{n}=\overline{\cup_{\alpha \in(0,1]}\left[L_{n, \alpha}, R_{n, \alpha}\right]}$ is compact. It is easy to verify that

$$
\begin{aligned}
& {\left[A_{l, \alpha}, A_{r, \alpha}\right] \subset\left[M_{1}, N_{1}\right],\left[B_{l, \alpha}, B_{r, \alpha}\right] \subset\left[M_{2}, N_{2}\right],} \\
& {\left[C_{l, \alpha}, C_{r, \alpha}\right] \subset\left[M_{3}, N_{3}\right],\left[L_{j, \alpha}, R_{j, \alpha}\right] \subset\left[M_{j}, N_{j}\right], j=-2,-1,0,}
\end{aligned}
$$

Where $A, B, C, x_{j}(j=-2,-1,0)$ are positive fuzzy numbers. It follows from (13) and (15) that 


$$
\left[L_{1, \alpha} R_{1, \alpha}\right] \subset\left[\frac{M_{1} M_{-2}}{N_{2}+N_{3} N_{-2} N_{-1} N_{0}}, \frac{N_{1} N_{-2}}{M_{2}+M_{3} M_{-2} M_{-1} M_{0}}\right], \alpha \in(0,1] .
$$

Moreover, one has

$$
\bigcup_{\alpha \in(0,1]}\left[L_{1, \alpha} R_{1, \alpha}\right] \subset\left[\frac{M_{1} M_{-2}}{N_{2}+N_{3} N_{-2} N_{-1} N_{0}}, \frac{N_{1} N_{-2}}{M_{2}+M_{3} M_{-2} M_{-1} M_{0}}\right], \alpha \in(0,1] .
$$

So, from (16), $\overline{\cup_{\alpha \in(0,1]}\left[L_{1, \alpha}, R_{1, \alpha}\right]}$ is compact. Therefore, it is evident that $\overline{\cup_{\alpha \in(0,1]}\left[L_{n, \alpha}, R_{n, \alpha}\right]}$ is compact by mathematical induction method.

Therefore from Lemma 3.2, relation (12), (14), and $L_{n, \alpha}, R_{n, \alpha}$ are left continuous, it means that $\left[L_{n, \alpha}, R_{n, \alpha}\right]$ determines a sequence of positive fuzzy numbers $\left\{x_{n}\right\}$ such that Eq. (6) holds.

Furthermore, it is proved that $\left\{x_{n}\right\}$ is the solution of equation (6) with initial conditions $x_{-2}, x_{-1}, x_{0}$. Since for all $\alpha \in(0,1]$

$$
\begin{gathered}
{\left[x_{n+1}\right]_{\alpha}=\left[L_{n+1, \alpha}, R_{n+1, \alpha}\right]} \\
=\left[\frac{A_{l, \alpha} L_{n-2, \alpha}}{B_{r, \alpha}+C_{r, \alpha} R_{n-2, \alpha} R_{n-1, \alpha} R_{n, \alpha}}, \frac{A_{r, \alpha} R_{n-2, \alpha}}{B_{l, \alpha}+C_{l, \alpha} L_{n-2, \alpha} L_{n-1, \alpha} L_{n, \alpha}}\right] \\
=\left[\frac{A x_{n-2}}{B+C x_{n-2} x_{n-1} x_{n}}\right]_{\alpha} .
\end{gathered}
$$

It is obvious that $\left\{x_{n}\right\}$ is the solution of Eq. (6) with initial conditions $x_{-2}, x_{-1}, x_{0}$.
Assume that there exists another solution $\left\{x_{n}^{*}\right\}$ of Eq. (6) with initial conditions $x_{-2}, x_{-1}, x_{0}$, then

$$
\left[x_{n}^{*}\right]_{\alpha}=\left[L_{n, \alpha}, R_{n, \alpha}\right], \alpha \in(0,1], n=0,1, \cdots
$$

It follows from (10) and (17) that

$$
\left[x_{n}\right]_{\alpha}=\left[x_{n}^{*}\right], \alpha \in(0,1], n=-2,-1, \cdots,
$$

From which it holds $x_{n}=x_{n}^{*}, \alpha \in(0,1], n=-2,-1, \cdots$, and then the proof is completed.

Next, the primary goal is to investigate the asymptotic behavior of the equilibrium point of Eq. (6). From the above proof process, $x_{n}$ is the unique positive solution of Eq. (6) with the initial values $x_{-2}, x_{-1}, x_{0}$, thus, it holds that

$$
\left[x_{n}\right]_{\alpha}=\left[L_{n, \alpha}, R_{n, \alpha}\right], \alpha \in(0,1], n=0,1, \cdots .
$$

Hence, it is obvious that $\left(L_{n, \alpha}, R_{n, \alpha}\right)$ satisfies the systems (9). To conveniently study the asymptotic behavior of Eq. (6), according to the systems (9), the corresponding linearized form of the system is constructed as follows

$$
y_{n+1}=\frac{a y_{n-2}}{f+d z_{n-2} z_{n-1} z_{n}}, z_{n+1}=\frac{b z_{n-2}}{e+c y_{n-2} y_{n-1} y_{n}}, n=0,1, \cdots,
$$

Where the parameters $a, b, c, d, e, f$ and initial conditions $y_{-2}, y_{-1}, y_{0}, z_{-2}, z_{-1}, z_{0}$ are positive real constants. Obviously, the systems (18) have a unique solution $\left(y_{n}, z_{n}\right)$ from Lemma 2.1.

Now, the primary purpose is to prove that the solution

$$
\text { (i) } 0 \leq y_{n} \leq\left\{\begin{array}{l}
\left(\frac{a}{f}\right)^{k+1} y_{-2}, n=3 k+1, \\
\left(\frac{a}{f}\right)^{k+1} y_{-1}, n=3 k+2, \\
\left(\frac{a}{f}\right)^{k+1} y_{0}, n=3 k+3,
\end{array} \quad \text { (ii) } 0 \leq y_{n} \leq \leq\left\{\begin{array}{l}
\left(\frac{b}{e}\right)^{k+1} z_{-2}, n=3 k+1, \\
\left(\frac{b}{e}\right)^{k+1} z_{-1}, n=3 k+2, \\
\left(\frac{b}{e}\right)^{k+1} z_{0}, n=3 k+3 .
\end{array}\right.\right.
$$

Proof. Using the induction method to prove (19). This assertion is true for $k=0$. Suppose that (19) is true for $k=m$, then it follows that for $k=m+1$ $\left(y_{n}, z_{n}\right)$ of the system (18) is bounded.

Theorem 3.2 let $\left(y_{n}, z_{n}\right)$ be positive solution of system (18), then for all $k \geq 0$, 


$$
y_{n}=\left\{\begin{array}{l}
y_{3(m+1)+1} \leq \frac{a}{f} y_{3(m+1)-2}=\frac{a}{f} y_{3 m+1} \leq \frac{a}{f} \cdot\left(\frac{a}{f}\right)^{m+1} y_{-2}, n=3(m+1)+1, \\
y_{3(m+1)+2} \leq \frac{a}{f} y_{3(m+1)+1-2}=\frac{a}{f} y_{3 m+2} \leq \frac{a}{f} \cdot\left(\frac{a}{f}\right)^{m+1} y_{-1}, n=3(m+1)+2, \\
y_{3(m+1)+3} \leq \frac{a}{f} y_{3(m+1)+2-2}=\frac{a}{f} y_{3 m+3} \leq \frac{a}{f} \cdot\left(\frac{a}{f}\right)^{m+1} y_{0}, n=3(m+1)+3,
\end{array}\right.
$$

And

$$
z_{n}=\left\{\begin{aligned}
& z_{3(m+1)+1} \leq \frac{b}{e} y_{3(m+1)-2}=\frac{b}{e} y_{3 m+1} \leq \frac{b}{e} \cdot\left(\frac{b}{e}\right)^{m+1} y_{-2}, n=3(m+1)+1, \\
& z_{3(m+1)+2} \leq \frac{b}{e} y_{3(m+1)+1-2}=\frac{b}{e} y_{3 m+2} \leq \frac{b}{e} \cdot\left(\frac{b}{e}\right)^{m+1} y_{-1}, n=3(m+1)+2, \\
& z_{3(m+1)+3} \leq \frac{b}{e} y_{3(m+1)+2-2}=\frac{b}{e} y_{3 m+3} \leq \frac{b}{e} \cdot\left(\frac{b}{e}\right)^{m+1} y_{0}, n=3(m+1)+3 .
\end{aligned}\right.
$$

The proof is completed.

Furthermore, it is easy to know that the systems (18) has two equilibrium points

$$
\bar{X}_{1}=\left(\bar{y}_{1}, \bar{z}_{1}\right)=(0,0), \bar{X}_{2}=\left(\bar{y}_{2}, \bar{z}_{2}\right)=\left(\left(\frac{b-e}{c}\right)^{1 / 3},\left(\frac{a-f}{d}\right)^{1 / 3}\right) \text {. }
$$

For the two equilibrium points, the following results are shown clearly.

Theorem 3.3 For the equilibrium point $\bar{X}_{1}=(0,0)$ of Eq.

$$
F\left(y_{n-2}, z_{n-2}, z_{n-1}, z_{n}\right)=\frac{a y_{n-2}}{f+d z_{n-2} z_{n-1} z_{n}}, G\left(z_{n-2}, y_{n-2}, y_{n-1}, y_{n}\right)=\frac{b z_{n-2}}{e+c y_{n-2} y_{n-1} y_{n}}
$$

Thus, one has

$$
\begin{aligned}
& F_{y_{n-2}}=\frac{a}{f+d z_{n-2} z_{n-1} z_{n}}, F_{z_{n-2}}=-\frac{a d y_{n-2} z_{n-1} z_{n}}{\left(f+d z_{n-2} z_{n-1} z_{n}\right)^{2}}, \\
& F_{z_{n-1}}=-\frac{a d y_{n-2} z_{n-2} z_{n}}{\left(f+d z_{n-2} z_{n-1} z_{n}\right)^{2}}, F_{z_{n}}=-\frac{a d y_{n-2} z_{n-2} z_{n-1}}{\left(f+d z_{n-2} z_{n-1} z_{n}\right)^{2}}, \\
& G_{z_{n-2}}=\frac{b}{e+c y_{n-2} y_{n-1} y_{n}}, G_{y_{n-2}}=-\frac{b c z_{n-2} y_{n-1} y_{n}}{\left(e+c y_{n-2} y_{n-1} y_{n}\right)^{2}}, \\
& G_{y_{n-1}}=-\frac{b c z_{n-2} y_{n-2} y_{n}}{\left(e+c y_{n-2} y_{n-1} y_{n}\right)^{2}}, G_{y_{n}}=-\frac{b c z_{n-2} y_{n-2} y_{n-1}}{\left(e+c y_{n-2} y_{n-1} y_{n}\right)^{2}} .
\end{aligned}
$$

Moreover, the linearized system of Eq. (18) about the equilibrium point $\bar{X}_{1}$ is given by

$$
\phi_{n+1}=D_{1} \phi_{n}
$$

Where (i) If $a<f, b<e$, then the equilibrium point $\bar{X}_{1}$ of the ystem (18) is unstable.

Proof. (i) Let $F:\left(R^{+}\right)^{3} \rightarrow R^{+}, G:\left(R^{+}\right)^{3} \rightarrow R^{+} \quad$ be multivariate function defined by system (18) is locally asymptotically stable, 
follows

$$
f(\lambda)=\frac{\left(a-f \lambda^{3}\right)\left(b-e \lambda^{3}\right)}{e f}=0
$$

Then roots of this characteristic equation are given by

$$
\lambda_{1,2,3}=\sqrt[3]{\frac{a}{f}}, \lambda_{4,5,6}=\sqrt[3]{\frac{b}{e}}
$$

Since all eigenvalues of the Jacobian matrix $D_{1}$ about the equilibrium point $\bar{X}_{1}$ lie in open unit disk $|\lambda|_{k}<1$, for all $k=1,2, \cdots, 6$. Hence from Lemma 2.2 the equilibrium point $\bar{X}_{1}$ is locally asymptotically stable.

(ii) Now, it is easy to see that if $a>f$ or $b>e$, then there Where

$$
\varphi_{n}=\left[\begin{array}{c}
y_{n} \\
y_{n-1} \\
y_{n-2} \\
z_{n} \\
z_{n-1} \\
z_{n-2}
\end{array}\right], D_{2}=\left[\begin{array}{cccccc}
0 & 0 & 1 & -\frac{d}{a} A_{1} B_{1} & -\frac{d}{a} A_{1} B_{1} & -\frac{d}{a} A_{1} B_{1} \\
1 & 0 & 0 & 0 & 0 & 0 \\
0 & 1 & 0 & 0 & 0 & 0 \\
-\frac{c}{b} A_{2} B_{2} & -\frac{c}{b} A_{2} B_{2} & -\frac{c}{b} A_{2} B_{2} & 0 & 0 & 1 \\
0 & 0 & 0 & 1 & 0 & 0 \\
0 & 0 & 0 & 0 & 1 & 0
\end{array}\right] .
$$

The characteristic equation of Eq. (22) is given by

$$
f(\lambda)=\frac{\left(\lambda^{2}+\lambda+1\right)^{2}\left(a b \lambda^{2}-2 a b \lambda+a b-A_{1} A_{2} B_{1} B_{2} c d\right)}{a b}=0,
$$

then roots of this characteristic equation are shown as follows

$$
\begin{aligned}
& \lambda_{1,2}=-\frac{1+\sqrt{3} i}{2}, \lambda_{3,4}=-\frac{-1+\sqrt{3} i}{2}, \\
& \lambda_{5}=1+\sqrt{\frac{A_{1} A_{2} B_{1} B_{2} c d}{a b}}=1+\sqrt{\frac{(b-e)(a-f)}{a b}}, \\
& \lambda_{6}=1-\sqrt{\frac{A_{1} A_{2} B_{1} B_{2} c d}{a b}}=1-\sqrt{\frac{(b-e)(a-f)}{a b}} .
\end{aligned}
$$

characteristic equation (23) such that $|\lambda|>1$. Therefore, If $a>f, b>e$ then the equilibrium point $\bar{X}_{2}$ of the system (18) is unstable. The proof is completed.

Lemma 3.3 [12] Let $I_{x}, I_{y}$ be some intervals of real numbers and assume that $f: I_{x}^{k+1} \times I_{y}^{l+1} \rightarrow I_{x} \quad$ and $g: I_{x}^{k+1} \times I_{y}^{l+1} \rightarrow I_{y}$ be continuously differential functions satisfying mixed monotone property. If there exists

Now, it is sufficient to prove that one of these roots has absolute value greater than one. It is obvious that if $a>f, b>e$, then there exists at least one root $\lambda$ of the

$$
\left\{\begin{array}{c}
m_{0} \leq \min \left\{x_{-k}, \cdots, x_{0}, y_{-l}, \cdots, y_{0}\right\} \leq \max \left\{x_{-k}, \cdots, x_{0}, y_{-l}, \cdots, y_{0}\right\} \leq M_{0} \\
n_{0} \leq \min \left\{x_{-k}, \cdots, x_{0}, y_{-l}, \cdots, y_{0}\right\} \leq \max \left\{x_{-k}, \cdots, x_{0}, y_{-l}, \cdots, y_{0}\right\} \leq N_{0}
\end{array}\right.
$$

Such that

$$
\begin{aligned}
& m_{0} \leq f\left(\left[m_{0}\right]_{p},\left[M_{0}\right]_{q},\left[n_{0}\right]_{s},\left[N_{0}\right]_{t}\right) \leq f\left(\left[M_{0}\right]_{p},\left[m_{0}\right]_{q},\left[N_{0}\right]_{s},\left[n_{0}\right]_{t}\right) \leq M_{0}, \\
& n_{0} \leq g\left(\left[m_{0}\right]_{p_{1}},\left[M_{0}\right]_{q_{1}},\left[n_{0}\right]_{s_{1}},\left[N_{0}\right]_{t_{1}}\right) \leq g\left(\left[M_{0}\right]_{p_{1}},\left[m_{0}\right]_{q_{1}},\left[N_{0}\right]_{s_{1}},\left[n_{0}\right]_{t_{1}}\right) \leq N_{0},
\end{aligned}
$$


Then there exist $(m, M) \in\left[m_{0}, M_{0}\right]^{2}$ and $(n, N) \in\left[n_{0}, N_{0}\right]^{2}$ satisfying

$$
\left\{\begin{array}{c}
M=f\left([M]_{p},[m]_{q},[N]_{s},[n]_{t}\right), m=f\left([m]_{p},[M]_{q},[n]_{s},[N]_{t}\right), \\
N=g\left([M]_{p_{1}},[m]_{q_{1}},[N]_{s_{1}},[n]_{t_{1}}\right), n=g\left([m]_{p_{1}},[M]_{q_{1}},[n]_{s_{1}},[N]_{t_{1}}\right) .
\end{array}\right.
$$

Moreover, if $m=M, n=N$, then the equations (7) has a unique equilibrium point $(\bar{x}, \bar{y}) \in\left[m_{0}, M_{0}\right] \times\left[n_{0}, N_{0}\right]$ and every solution of (7) converges to $(\bar{x}, \bar{y})$.

Theorem 3.5 If $a=b<e=f, c=d$, then the equilibrium point $\bar{X}_{1}=(0,0)$ of the system (18) is global attractor.

Proof. In view of $a=b<e=f, c=d$, the system (19) is changed to

$$
\begin{aligned}
y_{n+1} & =\frac{a y_{n-2}}{f+d z_{n-2} z_{n-1} z_{n}}, \\
z_{n+1} & =\frac{a z_{n-2}}{f+d y_{n-2} y_{n-1} y_{n}}, n=0,1, \cdots .
\end{aligned}
$$

Let $(f, g):(0, \infty)^{3} \times(0, \infty)^{3} \rightarrow(0, \infty) \times(0, \infty)$ be a function

$$
\begin{aligned}
& f_{u}=\frac{a}{f+d v w s}>0, f_{v}=-\frac{a d u w s}{(f+d v w s)^{2}}<0, \\
& f_{w}=-\frac{a d u v s}{(f+d v w s)^{2}}<0, f_{s}=-\frac{a d u v w}{(f+d v w s)^{2}}<0, \\
& g_{u^{*}}=\frac{a}{f+d v^{*} w^{*} s^{*}}>0, g_{v^{*}}=-\frac{a d u^{*} w^{*} s^{*}}{\left(f+d v^{*} w^{*} s^{*}\right)^{2}}<0, \\
& g_{w^{*}}=-\frac{a d u^{*} v^{*} s^{*}}{\left(f+d v^{*} w^{*} s^{*}\right)^{2}}<0, g_{s^{*}}=-\frac{a d u^{*} v^{*} w^{*}}{\left(f+d v^{*} w^{*} s^{*}\right)^{2}}<0,
\end{aligned}
$$

Which implies that $f$ and $g$ have a mixed monotone property.

$$
\begin{aligned}
& \text { Let } M_{0}=N_{0}=\max \left\{y_{-2}, y_{-1}, y_{0}, z_{-2}, z_{-1}, z_{0}\right\}, \\
& \sqrt[3]{(a-f) / d}<n_{0}=m_{0}<0 \text {, then } \\
& m_{0} \leq \frac{a m_{0}}{f+d N_{0}^{3}} \leq \frac{a M_{0}}{f+d n_{0}^{3}} \leq M_{0}, n_{0} \leq \frac{a n_{0}}{f+d M_{0}^{3}} \leq \frac{a N_{0}}{f+d m_{0}^{3}} \leq N_{0} .
\end{aligned}
$$

Obviously, since $m_{i}=n_{i}, M_{i}=N_{i}, i=0,1, \cdots$. then from the system (18) and Lemma 3.3, there exist $m, M \in\left[m_{0}, M_{0}\right], n=m, N=M$ such that

$$
m=\frac{a m}{f+d N^{3}}, n=\frac{a n}{f+d M^{3}}, M=\frac{a M}{f+d n^{3}}, N=\frac{a N}{f+d m^{3}},
$$

Then $M=m, N=n$.

Hence, it is proved that the equilibrium point $(0,0)$ of the system (18) is global attractor from Lemma 3.3. The proof is completed.

Moreover, from Definition 2.5 it follows that $\bar{X}_{1}$ is asymptotically stable.

Finally, the stability of the trivial solution of the fuzzy difference equation (6) will be discussed. Firstly, the following definition is introduced.

Definition 3.1[17] The trivial solution $x=\hat{0}$ of Eq. (6) is said to be stable, if given $\varepsilon>0$, there exists a $\delta(\varepsilon)>0$ with $D\left(x_{i}, \hat{0}\right)<\delta, i=-2,-1,0$, implies $D\left(x_{n}, \hat{0}\right)<\varepsilon$ for any $n>0$, such that for any $x_{i} \in D_{\delta}, i=-2,-1,0$, the solution $x_{n} \in D_{\varepsilon}, n>0$; attractive if there is a $\delta>0$ such that $D\left(x_{i}, \hat{0}\right)<\delta, i=-2,-1,0$, one has

$$
\lim _{n \rightarrow \infty} D\left(x_{n}, \hat{0}\right)=0
$$

(iii) asymptotically stable if (i) and (ii) hold simultaneously. Theorem 3.6 If the parameters $A, B, C$ are positive real numbers and $A<B$, then the trivial solution $x=\hat{0}$ of Eq. (6) is asymptotically stable with respect to $D$ when the initial conditions are positive fuzzy numbers with $\left[x_{i}\right]_{\alpha} \subset(0,+\infty), i=-2,-1,0, \alpha \in(0,1]$. 
Proof. The result follows from Theorem 3.3 and Theorem 3.5 .

\section{Numerical Simulation}

In this section a numerical example is given in order to support our theoretical results. The example represents the asymptotically behavior of solutions for the fuzzy difference system (6).

Example 4.1 Consider the following fuzzy difference equation

$$
x_{n+1}=\frac{A x_{n-2}}{B+C x_{n-2} x_{n-1} x_{n}}, n=0,1,2, \ldots,
$$

where $A, B, C$ are positive trivial fuzzy numbers. By Theorem 3.6, let

$$
\begin{gathered}
{[A]_{\alpha}=[A, A]=0.01,} \\
{[B]_{\alpha}=[B, B]=4,[C]_{\alpha}=[C, C]=2, \alpha \in(0,1] .}
\end{gathered}
$$

In addition, from Theorem 3.6, we denoted the initial conditions $x_{-2}, x_{-1}, x_{0}$ with $\left[x_{i}\right]_{\alpha} \subset(0,+\infty), i=-2,-1,0$, $\alpha \in(0,1]$ Such that

$$
x_{0}(x)=\left\{\begin{array}{l}
\frac{1}{3} x-\frac{5}{3}, 5 \leq x \leq 8, \\
-\frac{1}{5} x+\frac{13}{5}, 8 \leq x \leq 13,
\end{array} x_{-1}(x)=\left\{\begin{array}{l}
\frac{1}{4} x-\frac{1}{4}, 1 \leq x \leq 5, \\
-x+6,5 \leq x \leq 6,
\end{array} x_{-2}(x)=\left\{\begin{array}{c}
\frac{1}{5} x-\frac{2}{5}, 2 \leq x \leq 7, \\
-\frac{1}{3} x+\frac{10}{3}, 7 \leq x \leq 10 .
\end{array}\right.\right.\right.
$$

In view of (29),

$$
\left[x_{0}\right]_{\alpha}=[5+3 \alpha, 13-5 \alpha],\left[x_{-1}\right]_{\alpha}=[4+4 \alpha, 6-\alpha],\left[x_{-2}\right]_{\alpha}=[2+5 \alpha, 10-3 \alpha] \text {. }
$$

Moreover, from Eq. (28), a coupled system of difference equation with parameter $\alpha$ is obtained

$$
\begin{aligned}
& L_{n+1, \alpha}=\frac{0.01 L_{n-2, \alpha}}{4+2 R_{n-2, \alpha} R_{n-1, \alpha} R_{n, \alpha}}, \\
& R_{n+1, \alpha}=\frac{0.01 R_{n-2, \alpha}}{4+2 L_{n-2, \alpha} L_{n-1, \alpha} L_{n, \alpha}}, \alpha \in(0,1], n=0,1, \cdots
\end{aligned}
$$

It is easy to prove the conditions of Theorem 3.6 is satisfied. So from Theorem 3.6 the trivial solution $x=\hat{0}$ of Eq. (6) is asymptotically stable with respect to $D$ as $n \rightarrow \infty$ (see Figureures 1-6).

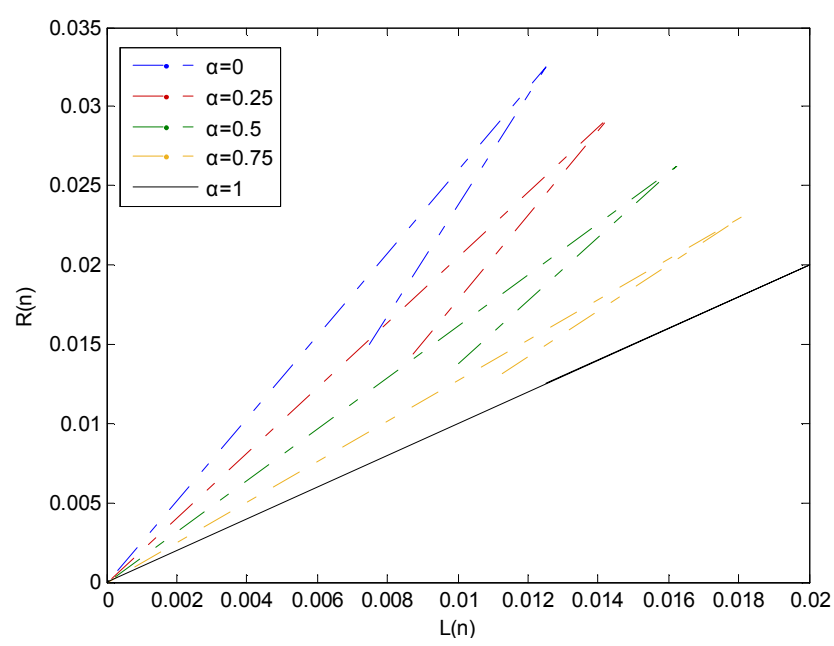

Figure 1. The dynamics of system (30).

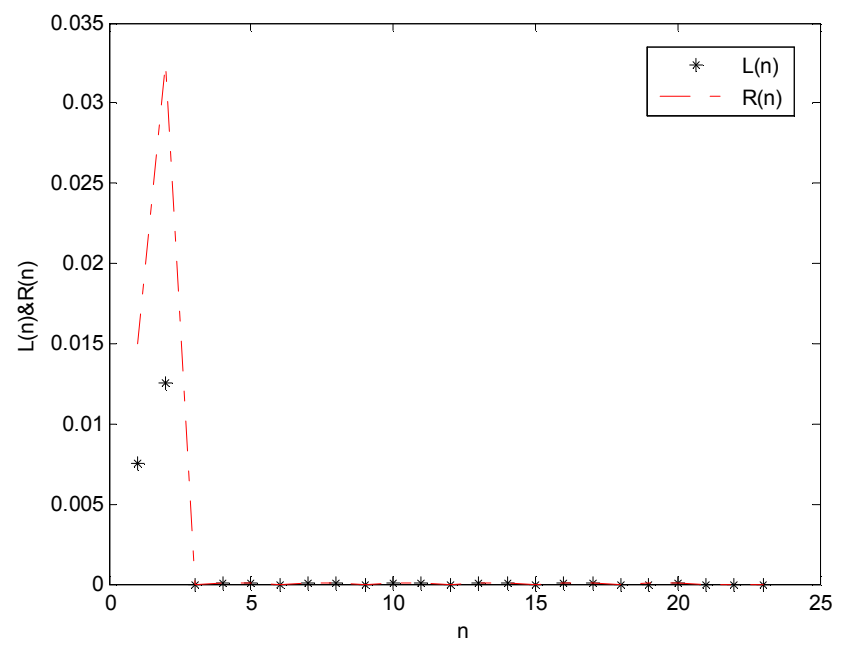

Figure 2. The solution of system (30) when $\alpha=0$.

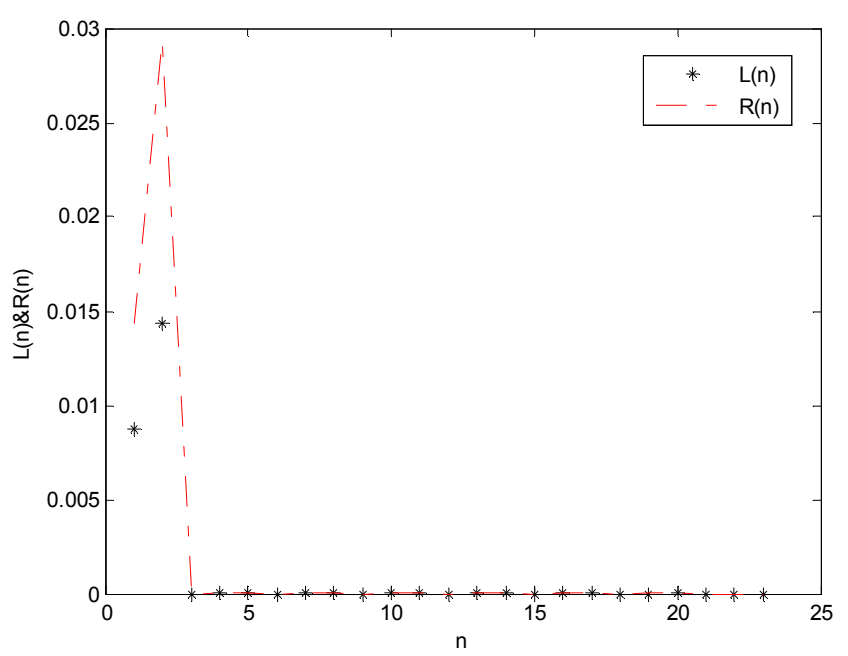

Figure 3. The solution of system (30) when $\alpha=0.25$. 


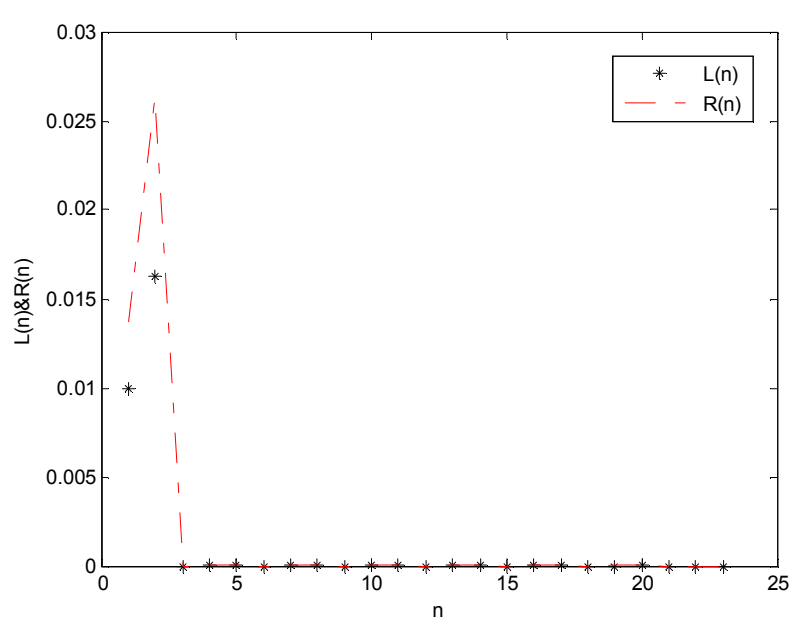

Figure 4. The solution of system (30) when $\alpha=0.5$.

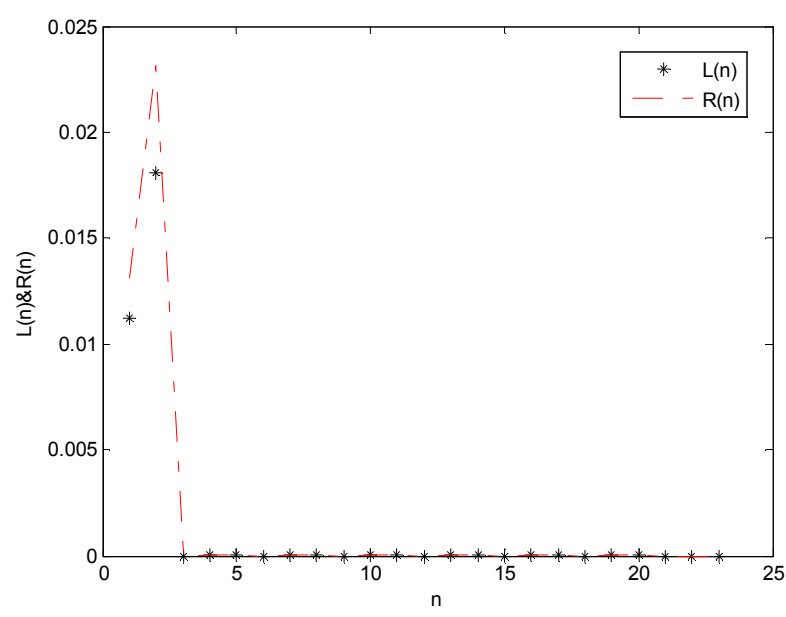

Figure 5. The solution of system (30) when $\alpha=0.75$.

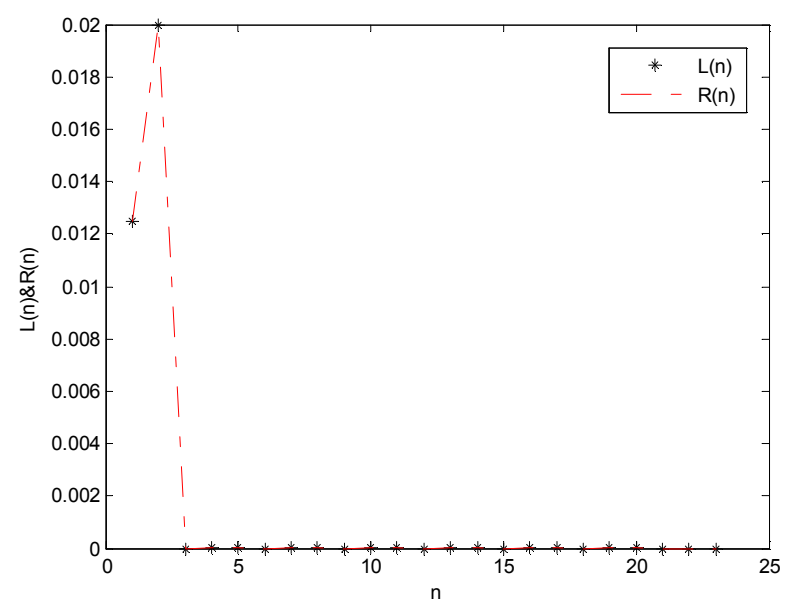

Figure 6. The solution of system (30) when $\alpha=1$.

\section{Conclusion}

The main purpose of this paper is to deal with the dynamics behavior for a class of nonlinear third-order fuzzy difference equations. Firstly, the existence and uniqueness of positive fuzzy solutions is proved. Secondly, It is obtained that the nonzero equilibrium points of the corresponding ordinary difference equations is unstable by using linearization method. Finally, It is find that the zero trivial solution of the fuzzy equation is stable when the parameters $A, B, C$ are positive real numbers, $A<B$ and the initial conditions are any positive fuzzy numbers. In particular, an example is given to show the effectiveness of the obtained results. In addition, the sufficient conditions obtained in this paper are very simple, which provide flexibility for the application and analysis of nonlinear fuzzy difference equation. For further work, it is our next research target to study the higher order fuzzy difference equations using new iteration method, inequality skills and comparison theorem.

\section{Acknowledgements}

This work is supported the Sichuan Science and Technology Program (Grant no. 2018JY0480) of China, the National Nature Science Fund (Project no. 61503053) of China, the Natural Science Foundation Project of CQ CSTC (Grant no. cstc2015jcyj BX0135) of China.

\section{References}

[1] M. S. Guo , X. P. Xue, R. L. Li, Impulsive functional differential inclusions and fuzzy population models, Fuzzy Sets and Systems, 138 (2003) 601-615.

[2] E. Y. Deeba, A. De Korvin, Analysis by fuzzy difference equations of a model of $\mathrm{CO} 2$ level in the blood, Applied Mathematics Letters, 12 (3) (1999) 33-40.

[3] E. C. Pielou, Population and community ecology: principles and methods, CRC Press, London, (1974).

[4] E. P. Popov, Automatic regulation and control, (Russian) Nauka, Moscow, (1966).

[5] K. A. Chrysafis, B. K. Papadopoulos, G. Papaschinopoulos, On the fuzzy difference equations of finance, Fuzzy Sets and Systems, 159 (24) (2008) 3259- 3270.

[6] J. P. Sun, W. T. Li, Multiple positive solutions of a discrete difference system, Applied Mathematics and Computation, 143 (2-3) (2003) 213-221.

[7] Z. AISharawi, Periodic orbits in periodic discrete dynamics, Computers and Mathematics with Applications, 56 (8) (2008) 1966-1974.

[8] R. DeVault, G. Ladas, S. W. Schultz, On the recursive sequence $x_{n+1}=A / x_{n}+1 / x_{n-2}$, Proceedings of the American Mathematical Society,126 (11) (1998) 3257-3261.

[9] G. Papaschinopoulos, C. J. Schinas, On a system of two nonlinear difference equation, Journal of Mathematical Analysis and Applications, 219 (2) (1998) 415-426.

[10] Q. H. Zhang, L. H. Yang, D. X. Liao, On Fuzzy Difference Equation $x_{n+1}=\left(a+b x_{n}\right) /\left(A+x_{n-1}\right) \quad$ Fuzzy Systems and Mathematics, 26 (4) (2012) 99-107.

[11] Q. H. Zhang, L. H. Yang, D. X. Liao, On first order fuzzy Ricatti difference equation, Information Sciences, 270 (20) (2014) 226-236. 
[12] C.Y.Wang, X. L. Su, P. Liu, X. H. Hu, R. Li, On the dynamics of a five-order fuzzy difference equation, Journal of Nonlinear Sciences and Applications, 10 (6) (2017) 3303-3319.

[13] A. Khastan, New solutions for first order linear fuzzy difference equations, Journal of Computational and Applied Mathematics, 312 (2017) 156-166.

[14] E. Y. Deeba, A. De Korvin, E. L. Koh, A fuzzy difference equation with an application, Journal of Difference Equations and Applications, 2 (4) (1996) 365- 374.

[15] G. Stefanidou, G. Papaschinopoulos, A fuzzy difference equation of a rational form, Journal of Nonlinear Mathematical Physics, 12 (2) (2005) 300-315.

[16] S. J. Song, L. Guo, C. B. Feng, Global existence of solutions to fuzzy differential equations, Fuzzy Sets and Systems, 115 (3) (2000) 371-376.

[17] V. Lakshmikantham, A. S. Vatsala, Basic theory of fuzzy difference equations, Journal of Difference Equations and Applications, 8 (11) (2002) 957-968.

[18] Q. H. Zhang, J. Z. Liu, Z. G. Luo, Dynamical behavior of a third-order rational fuzzy difference equation, Advances in Difference Equations, 2015 (2015), Article ID: 108.

[19] W. T. Li, H. R. Sun, Dynamics of a rational difference equation, Applied Mathematics and Computation, 163 (2) (2005) 577-591.

[20] T. X. Sun, H. J. Xi, L. Hong, On the system of rational difference equations $x_{n+l}=f\left(x_{n}, y_{n-k}\right), y_{n+l}=f\left(y_{n}, x_{n-k}\right)$ Advances in Difference Equations, 2006 (2006), Article ID: 16949.

[21] Q. Din, MN. Qureshi, A. Q. Khan, Dynamics of a fourth-order system of rational difference equations, Advances in Difference Equations, 2012 (2012), Article ID: 215.

[22] Q. H. Zhang, L. H. Yang, J. Z. Liu, Dynamics of a system of rational third-order difference equation, 2012 (2012), Article ID: 136 .

[23] S. Stević, Global stability and asymptotics of some classes of rational difference equations, Journal of Mathematical Analysis and Applications, 316 (1) (2006) 60-68.

[24] C. Y. Wang, S. Wang, W. Wang, Global asymptotic stability of equilibrium point for a family of rational difference equations. Applied Mathematics Letters, 24 (2011) 714-718.

[25] P. Liu, C. Y. Wang, Yonghong Li, Rui Li, Asymptotic behavior of equilibrium point for a system of fourth-order rational difference equations, Journal of Computational Analysis and Applications, 27(6) (2019) 947-961.

[26] C. Y. Wang, Y. Q. Zhou, S. Pan, R. Li, On a system of three max-type nonlinear difference equations, Journal of Computational Analysis and Applications, 25(8) (2018) 1463-1479.

[27] C. Y. Wang, X. J. Fang, R. Li, On the dynamics of a certain four-order fractional difference equations, Journal of Computational Analysis and Applications, 22(5) (2017) 968-976.

[28] C. Y. Wang, X. T. Jing, X. H. Hu, R. Li, On the periodicity of a max-type rational difference equation, Journal of Nonlinear Sciences and Applications, 10(9) (2017) 4648-4661.

[29] C. Y. Wang, H. Liu, R. Li, X. H. Hu, Y.B. Shao, Boundedness character of a symmetric system of max-type difference equations, IAENG International Journal of Applied Mathematics, 46(4) (2016) 505-511.

[30] C. Y. Wang, X. J. Fang, R. Li, On the solution for a system of two rational difference equations, Journal of Computational Analysis and Applications, 2016, 20(1) (2016) 175-186.

[31] B. Bede, Mathematics of fuzzy sets and fuzzy logic, Springer, London, (2013).

[32] P. Diamond, P. Kloeden, Metric Spaces of Fuzzy Sets, World Scientific, Singapore, (1994).

[33] V. L. Kocic, G. Ladas, Global Behavior of Nonlinear Difference Equations of Higher Order with Applications, Kluwer Academic, Dordrecht, (1993).

[34] H. Sedaghat, Nonlinear Difference Equations: Theory with Applications to Social Science Models, Kluwer Academic Publishers, Dordrecht, (2003).

[35] E. Camouzis, G. Ladas, Dynamics of third-order rational difference equations with open problems and conjectures, Chapman and Hall/HRC, Boca Raton, FL, (2008).

[36] G. Papaschinopoulos, B. K. Papadopoulos, On the fuzzy difference equation $x_{n+l}=A+x_{n} / x_{n-m}$ Fuzzy Sets and Systems, 129 (1) (2002) 73-81.

[37] C. X. Wu, B. K. Zhang, Embedding problem of noncompact fuzzy number space $E$ (I), Fuzzy Sets and Systems, 105 (1) (1999) 165-169. 